\title{
How fructophilic lactic acid bacteria may reduce the FODMAPs content in wheat-derived baked goods: a proof of concept
}

\author{
Marta Acín Albiac ${ }^{1}$, Raffaella Di Cagno ${ }^{1^{*}}$ (D) Pasquale Filannino ${ }^{2^{*}}$, Vincenzo Cantatore ${ }^{2}$ and Marco Gobbetti ${ }^{1}$
}

\begin{abstract}
Background: FODMAPs (Fermentable oligosaccharides, disaccharides, monosaccharides, and polyols) intake is associated with the onset of irritable bowel syndrome symptoms. FODMAPs in wheat-derived baked goods may be reduced via bioprocessing by endogenous enzymes and/or microbial fermentation. Because of the inherent enzyme activities, bread made by baker's yeast and sourdough may result in decreased levels of FODMAPs, whose values are, however, not enough low for people sensitive to FODMAPs.

Results: Our study investigated the complementary capability of targeted commercial enzymes and metabolically strictly fructophilic lactic acid bacteria (FLAB) to hydrolyze fructans and deplete fructose during wheat dough fermentation. FLAB strains displayed higher fructose consumption rate compared to conventional sourdough lactic acid bacteria. Fructose metabolism by FLAB was faster than glucose. The catabolism of mannitol with the goal of its reuse by FLAB was also investigated. Under sourdough conditions, higher fructans breakdown occurred in FLAB inoculated doughs compared to conventional sourdough bacteria. Preliminary trials allowed selecting Apilactobacillus kunkeei B23I and Fructobacillus fructosus MBIII5 as starter candidates, which were successfully applied in synergy with commercial invertase for low FODMAPs baking.
\end{abstract}

Conclusions: Results of this study clearly demonstrated the potential of selected strictly FLAB to strongly reduce FODMAPs in wheat dough, especially under liquid-dough and high oxygenation conditions.

Keywords: FODMAP, IBS, Fructophilic lactic acid bacteria, Fructans, Wheat flour, Bread

\section{Background}

Fermentable oligosaccharides, disaccharides, monosaccharides, and polyols (FODMAPs) are heterogeneous compounds, which, due to their chemical structure, are poorly digestible and may exert a range of effects on human gastrointestinal process. Fructans, galacto-oligosaccharides (GOS), lactose, fructose in excess of glucose, and sugar polyols (sorbitol and mannitol) are the main FODMAPs found in foods $[1,2]$. Whether some

\footnotetext{
*Correspondence: raffaella.dicagno@unibz.it; pasquale.filannino1@uniba.it

${ }^{1}$ Faculty of Sciences and Technology, Libera Università di Bolzano,

39100 Bolzano, Italy

${ }^{2}$ Department of Soil, Plant and Food Science, University of Bari Aldo Moro, 70126 Bari, Italy
}

FODMAPs are considered prebiotics contributing to the healthy maintenance of intestinal microbiota, on the other side, FODMAPs intake is associated with the onset of irritable bowel syndrome (IBS) symptoms and other functional gut disorders $[1,2]$. IBS is the most common gastrointestinal disorder worldwide, with a prevalence estimated between 7 and $21 \%$ of the global population [2]. Triggering of IBS symptoms following consumption of FODMAPs is due to their poor absorption at the small intestine level. Some FODMAPs are osmotically active, and their malabsorption draws water into the intestinal and/or colonic lumen, causing diarrhea. Once reached the distal ileum and colon, FODMAPs undergo the fermentation to short-chain 
fatty acids and gases, causing bloating, abdominal pain, and flatulence $[1,2]$. FODMAPs may also trigger the manifestation of non-celiac wheat sensitivity [3, 4]. A low-FODMAPs diet may reduce symptoms associated to IBS or non-celiac wheat sensitivity $[5,6]$. Being FODMAPs responsible for diversified effects along the gastrointestinal tract, a cutoff value for each compound was definable, which ranges from 0.15 to $1 \mathrm{~g}$ per serving/meal for IBS patients [7]. Anyway, a diet therapy based on the avoidance of FODMAPs restricts too much the food choices, eliminating staple foods, likely wheat derivatives and various vegetables, legumes and fruits. Under this dietary regimen, IBS patients should suffer for reduced intakes of fiber, minerals, vitamins, and phytochemicals. In addition to the unbalanced nutritional status, the drastic reduction of FODMAPs interferes with the intestinal microbiota and colonocyte metabolism [8].

Wheat-derived baked goods account for the major part of the daily consumed FODMAPs, mainly represented by fructans and, to a lesser extent, GOS and fructose, whereas mannitol is mostly present in sourdough bread [9]. Considering the risks associated to prolonged implementation of low-FODMAP diet, various biotechnology strategies are under investigation for the manufacture of wheat-derived baked goods with reduced FODMAPs content and suitable for IBS patients [10]. For instance, the use enzymes degrading fructans lowered the level of FODMAPs in bread. Whether humans lack these enzymes, they are common in many plants and microorganisms. Exoinulinases (EC 3.2.1.80) are specific for the terminal linkages of inulin and release fructose, whereas endoinulinases (EC 3.2.1.7) have as target the internal $\beta(2-1)$ glycosidic linkage, resulting into oligosaccharides with a lower degree of polymerization. Using an exo-mechanism, invertase ( $\beta$-fructofuranoside fructohydrolase EC 3.2.1.26) is able to catalyze the degradation of inulin [10]. Apart from the enzyme used, the hydrolysis of fructans correlates with the release of fructose, which is also within the FODMAP group, but only when present in excess to glucose. Indeed, when fructose is present at the luminal level together with equimolar amounts of free glucose, the apical GLUT2 transporter prevents the risk of fructose malabsorption [11]. Because of the inherent enzyme activities, bread making by baker's yeast and sourdough may result in decreased levels of FODMAPs, whose values are, however, not enough low for people sensitive to FODMAPs. Several causes might be responsible for this unsatisfactory result, likely the short leavening/fermentation time, the release of fructose from fructans, and the capability of hetero-fermentative lactic acid bacteria to convert the fructose to mannitol [10]. Therefore, low FODMAP baking needs dedicated approaches targeting fructans-, fructose-, and mannitoldegrading enzymes/organisms [9].

Our study aimed at investigating the complementary capability of targeted commercial enzymes and strictly fructophilic lactic acid bacteria (FLAB) to fully degrade FODMAPs during wheat dough fermentation. As a newly discovered bacterial group, FLAB are gaining increasing interest for their potential applications in food and pharmaceutical field [12]. First, we screened strictly FLAB for their fructose consumption rates and mannitol degrading capabilities. Besides, we developed a novel protocol applied both under firm- and liquid-dough fermentation [13].

\section{Results}

Fructose consumption rate by FLAB during growth into fructose-glucose based medium

Fructose consumption rate by FLAB strains, as well by Lactiplantibacillus plantarum DC400 (formerly Lactobacillus plantarum) and Fructilactobacillus sanfranciscensis SD8 (formerly Lactobacillus sanfranciscensis) (controls), was investigated during growth into Fructose Glucose Yeast extract Peptone (FGYP) broth under aerobic conditions. After $24 \mathrm{~h}$ at $30{ }^{\circ} \mathrm{C}$, all the strains reached a cell density of ca. 9-9.5 $\mathrm{Log} \mathrm{CFU} \mathrm{ml}{ }^{-1}$. The fructose available in the medium $\left(10 \mathrm{~g} \mathrm{~L}^{-1}\right)$ was completely consumed after 12-14 h by all FLAB, with the exception of Apilactobacillus kunkeei PLB29 and PFA3 (formerly Lactobacillus kunkeei), and Fructobacillus fructosus PFA23, for which depletion was reached after 16 h. L. plantarum DC400 and F. sanfranciscensis SD8 slowly metabolized fructose; it was still detectable after $24 \mathrm{~h}$. Kinetics of fructose consumption were modeled according to the Gompertz equation as modified by Zwietering et al. [14] (Table 1). A. kunkeei strains showed $\mu_{\max }$ values ranging from $1.28 \pm 0.18$ to $3.51 \pm 0.10 \mathrm{~g} \mathrm{~L}^{-1} \mathrm{~h}^{-1}$, where $\lambda$ values ranged from $1.47 \pm 0.17$ to $8.32 \pm 0.28 \mathrm{~h}$. When Fructo. fructosus strains were used, $\mu_{\max }$ and $\lambda$ ranged from $0.96 \pm 0.15$ to $3.02 \pm 0.15 \mathrm{~g} \mathrm{~L}^{-1} \mathrm{~h}^{-1}$, and from $3.07 \pm 0.25$ to $5.32 \pm 0.32 \mathrm{~h}$, respectively. As expected, the lowest $(\mathrm{P}<0.05)$ values of $\mu_{\max }$ were found with $L$. plantarum DC400 $\left(0.28 \pm 0.14 \mathrm{~g} \mathrm{~L}^{-1} \mathrm{~h}^{-1}\right)$ and F. sanfranciscensis SD8 $\left(0.88 \pm 0.08 \mathrm{~g} \mathrm{~L}^{-1} \mathrm{~h}^{-1}\right)$. This latter had also the highest value of $\lambda(13.76 \pm 0.39 \mathrm{~h})$. On the other side, glucose metabolism by FLAB was slower than fructose (data not shown).

\section{Mannitol consumption by FLAB into a mannitol-based media}

Aiming at selecting the best performing FLAB strains to metabolize mannitol, they were grown on Mannitol Yeast extract Peptone (MYP) agar supplemented with $\mathrm{CaCO}_{3}$, containing mannitol as the only carbon source. Mannitol 
Table 1 Kinetics parameters $\left(\mu_{\max } \text { and } \lambda\right)^{*}$ of fructose consumption by bacterial strains

\begin{tabular}{|c|c|c|}
\hline Strain & $\mu_{\max }$ & $\lambda$ \\
\hline Apilactobacillus kunkeei BIII59 & $1.98 \pm 0.12^{\mathrm{fg}}$ & $3.05 \pm 0.12^{\prime}$ \\
\hline A. kunkeei BIII60 & $1.28 \pm 0.18^{\mathrm{ij}}$ & $3.36 \pm 0.24^{\mathrm{ijkl}}$ \\
\hline A. kunkeei BVI14 & $1.41 \pm 0.13^{i}$ & $2.99 \pm 0.29^{\prime}$ \\
\hline A. kunkeei B17 & $2.41 \pm 0.14^{\text {de }}$ & $8.32 \pm 0.28^{b}$ \\
\hline A. kunkeei B23I & $3.51 \pm 0.10^{\mathrm{a}}$ & $4.21 \pm 0.22^{f}$ \\
\hline A. kunkeei B7 & $1.81 \pm 0.19^{g h}$ & $3.06 \pm 0.121$ \\
\hline A. kunkeei BV61 & $1.87 \pm 0.14^{\mathrm{gh}}$ & $3.76 \pm 0.36^{\text {fghijk }}$ \\
\hline A. kunkeei BV20 & $2.05 \pm 0.13^{\mathrm{fg}}$ & $4.16 \pm 0.28 \mathrm{fg}$ \\
\hline A. kunkeei BVI52 & $2.79 \pm 0.08^{b c}$ & $3.84 \pm 0.24^{\mathrm{fghi}}$ \\
\hline A. kunkeei B4I & $1.89 \pm 0.07^{9 h}$ & $3.11 \pm 0.26^{\mathrm{jkl}}$ \\
\hline A. kunkeei PLB29 & $1.42 \pm 0.10^{i}$ & $3.65 \pm 0.21^{\text {ghij }}$ \\
\hline A. kunkeei PLA21 & $2.92 \pm 0.15^{b c}$ & $3.85 \pm 0.14^{\mathrm{fgh}}$ \\
\hline A. kunkeei PFA3 & $1.75 \pm 0.21^{\text {gh }}$ & $7.39 \pm 0.38^{c}$ \\
\hline A. kunkeei PLB20 & $1.86 \pm 0.22^{g h}$ & $3.50 \pm 0.25^{\text {hijk }}$ \\
\hline A. kunkeei PLB 34 & $2.57 \pm 0.19^{\text {cde }}$ & $5.64 \pm 0.36^{d}$ \\
\hline A. kunkeei PL24 & $1.50 \pm 0.24^{\mathrm{hi}}$ & $1.47 \pm 0.17^{m}$ \\
\hline A. kunkeei PF16 & $2.66 \pm 0.28^{\text {bcde }}$ & $3.43 \pm 0.22^{\text {hijkl }}$ \\
\hline A. kunkeei PF6 & $3.20 \pm 0.18^{b c}$ & $5.18 \pm 0.31^{\mathrm{de}}$ \\
\hline A. kunkeei BEE4 & $3.24 \pm 0.23^{\mathrm{ab}}$ & $7.46 \pm 0.27^{c}$ \\
\hline Fructobacillus fructosus MBIII5 & $3.02 \pm 0.15^{b c}$ & $5.30 \pm 0.33^{d}$ \\
\hline Fructo. fructosus B5 & $2.22 \pm 0.17^{\mathrm{ef}}$ & $3.83 \pm 0.28^{\text {fghi }}$ \\
\hline Fructo. fructosus MBIII2 & $2.26 \pm 0.19^{\mathrm{ef}}$ & $3.07 \pm 0.25^{\mathrm{kl}}$ \\
\hline Fructo. fructosus B1 & $1.73 \pm 0.22^{g h}$ & $4.78 \pm 0.24^{e}$ \\
\hline Fructo. fructosus PL21 & $2.82 \pm 0.17^{b c}$ & $5.32 \pm 0.32^{d}$ \\
\hline Fructo. fructosus PFA23 & $0.96 \pm 0.15^{\mathrm{jk}}$ & $3.29 \pm 0.31^{\mathrm{ijkl}}$ \\
\hline Lactiplantibacillus plantarum DC400 & $0.28 \pm 0.14^{1}$ & $0.22 \pm 0.08^{n}$ \\
\hline Fructilactobacillus sanfranciscensis SD8 & $0.88 \pm 0.08^{k}$ & $13.76 \pm 0.39^{a}$ \\
\hline
\end{tabular}

Fructose consumption was determined during growth of bacterial strains in FGYP broth at $30^{\circ} \mathrm{C}$ for $24 \mathrm{~h}$

*Kinetics were modelled according to the Gompertz equation as modified by Zwietering et al. [14]: $\mu_{\max }$ is maximum consumption rate (expressed as $\mathrm{gl}^{-1}$ $\left.\mathrm{h}^{-1}\right), \lambda$ is the length of the lag phase expressed in hours

${ }^{a-n}$ Values in the same column with different superscript letters are significantly different $(P<0.05)$

consumption was evaluated by measuring the diameter of the clearance zone surrounding the colonies. Only for six strains, a clearance zone was observed. Fructo. fructosus MBIII5 showed the widest $(\mathrm{P}<0.05)$ halo $(2.6 \pm 0.3 \mathrm{~cm})$, followed by Fructo. fructosus MBIII2 $(1.6 \pm 0.4 \mathrm{~cm})$ and B5 $(1.4 \pm 0.4 \mathrm{~cm})$, and A. kunkeei B7 $(1.3 \pm 0.7 \mathrm{~cm})$. The less extensive halos were found for Fructo. fructosus PL21 $(0.4 \pm 0.3 \mathrm{~cm})$ and PFA23 $(0.4 \pm 0.4 \mathrm{~cm})$. Based on the highest metabolic activity of Fructo. fructosus MBIII5 on MYP agar compared to the other strains, mannitol consumption by Fructo. fructosus MBIII5 was further investigated during growth into MYP broth containing mannitol $\left(10 \mathrm{~g} \mathrm{~L}^{-1}\right)$ as the only carbon source (Fig. 1). During $24 \mathrm{~h}$ of incubation, this strain metabolized

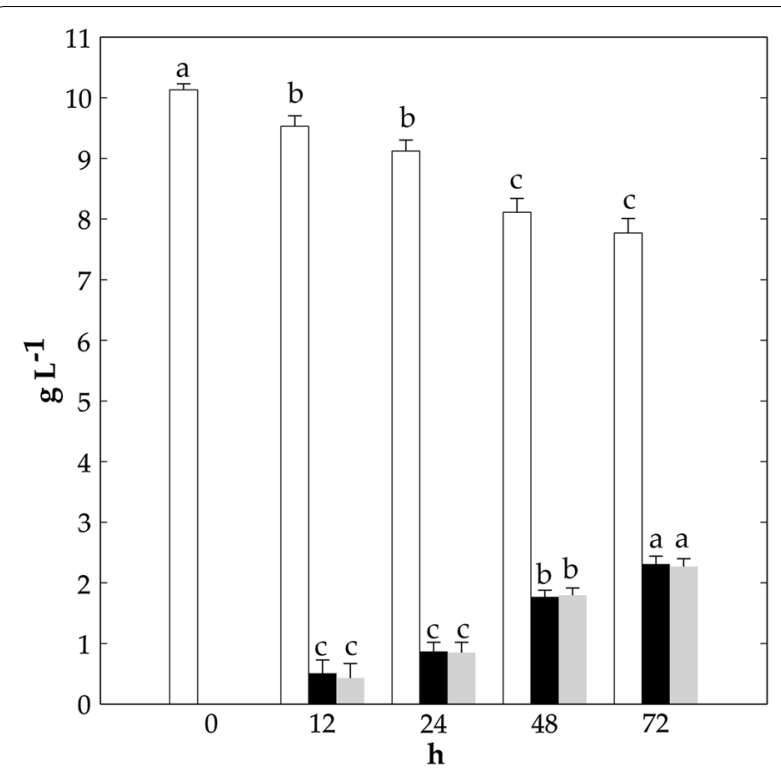

Fig. 1 Mannitol (white bars) and lactic (black bars) and acetic (grey bars) acids level $\left(\mathrm{g} \mathrm{L}^{-1}\right)$. Fructobacillus fructosus MBIII5 was grown in Mannitol Yeast extract Peptone (MYP) broth at $30^{\circ} \mathrm{C}$ for $72 \mathrm{~h}$ under aerobic condition in shaking (200 rpm) flasks with baffles For each compound, bars with different superscript letters $(\mathrm{a}-\mathrm{c})$ are significantly different $(P<0.05)$

mannitol $(1.02 \pm 0.10 \mathrm{~g} \mathrm{~L}-1)$ to lactic $(0.87 \pm 0.15 \mathrm{~g} \mathrm{~L}-1)$ and acetic acids $(0.85 \pm 0.17 \mathrm{~g} \mathrm{~L}-1)$. Mannitol was further metabolized after $48(2.03 \pm 0.14 \mathrm{~g} \mathrm{~L}-1)$ and $72 \mathrm{~h}$ $(2.36 \pm 0.15 \mathrm{~g} \mathrm{~L}-1)$ of incubation (Fig. 1).

\section{Growth and acidification performances of the selected FLAB strains under sourdough conditions}

Based on the kinetics of fructose consumption that showed the highest value of $\mu_{\max }\left(3.51 \pm 0.10 \mathrm{~g} \mathrm{~L}^{-1} \mathrm{~h}^{-1}\right)$, A. kunkeei B23I was selected as starter for sourdough fermentation (DY 160). Fructo. fructosus MBIII5 was also selected for its capability to use mannitol as carbon source. L. plantarum DC400 and F. sanfranciscensis SD8 were used as control starters. A control dough (DY 160) was prepared only with durum wheat flour and sterile tap water, without bacterial inoculum (unstarted dough) and incubated for $24 \mathrm{~h}$. From an initial cell density of ca. 7 Log CFU g ${ }^{-1}$, both selected FLAB strains grew by ca. 1.8 logarithmic cycles after $12 \mathrm{~h}$ at $30^{\circ} \mathrm{C}$. Cell density remained almost stable until 24 h. L. plantarum DC400 led to an increase of ca. 2 and $2.5 \log$ cycles after 12 and 24 h, respectively. F. sanfranciscensis SD8 grew by ca. 1.6 Log cycles after $12 \mathrm{~h}$ and by ca. 2 Log cycles after $24 \mathrm{~h}$. After 24 of incubation, the cell density of lactic acid bacteria into the unstarted dough was ca. $3 \mathrm{Log} \mathrm{CFU} \mathrm{g}^{-1}$.

The initial $\mathrm{pH}$ of the dough was $6.2 \pm 0.02$. After $12 \mathrm{~h}$, the lowest $(\mathrm{P}<0.05)$ value of $\mathrm{pH}$ was found for $L$. 
plantarum DC400 (4.08 \pm 0.03$)$, followed by A. kunkeei B23I (4.86 \pm 0.01$)$, Fructo. fructosus MBIII5 (5.04 \pm 0.02$)$ and F. sanfranciscensis SD8 $(5.01 \pm 0.02)$. Values of $\mathrm{pH}$ dropped further throughout the fermentation and reached the final values of $4.16-4.11$ with $A$. kunkeei B23I, Fructo. fructosus MBIII5, and F. sanfranciscensis SD8. The lowest $(\mathrm{P}<0.05)$ value was found for $L$. plantarum DC400 $(3.78 \pm 0.03)$. The $\mathrm{pH}$ values of the unstarted dough ranged from 6.2 to 5.75 .
Total fructans, fructose, glucose, and mannitol levels in fermented doughs

A. kunkeei B23I and Fructo. fructosus MBIII5 were used both as single starters and as binary combination to ferment wheat flour doughs (DY 160) for $24 \mathrm{~h}$ at $30{ }^{\circ} \mathrm{C}$. Doughs fermented by L. plantarum DC400 or F. sanfranciscensis SD8 were also analyzed. A dough without bacterial inoculums was used as control (unstarted dough). Total fructans, fructose, glucose, and mannitol were monitored after 12 and $24 \mathrm{~h}$ of fermentation. The initial concentration of total fructans was $1003 \pm 40 \mathrm{mg}$ per $100 \mathrm{~g}$ of flour. After $12 \mathrm{~h}$ only a slow decrease of fructans $(18-25 \%)$ was found in all fermented samples. After $24 \mathrm{~h}$
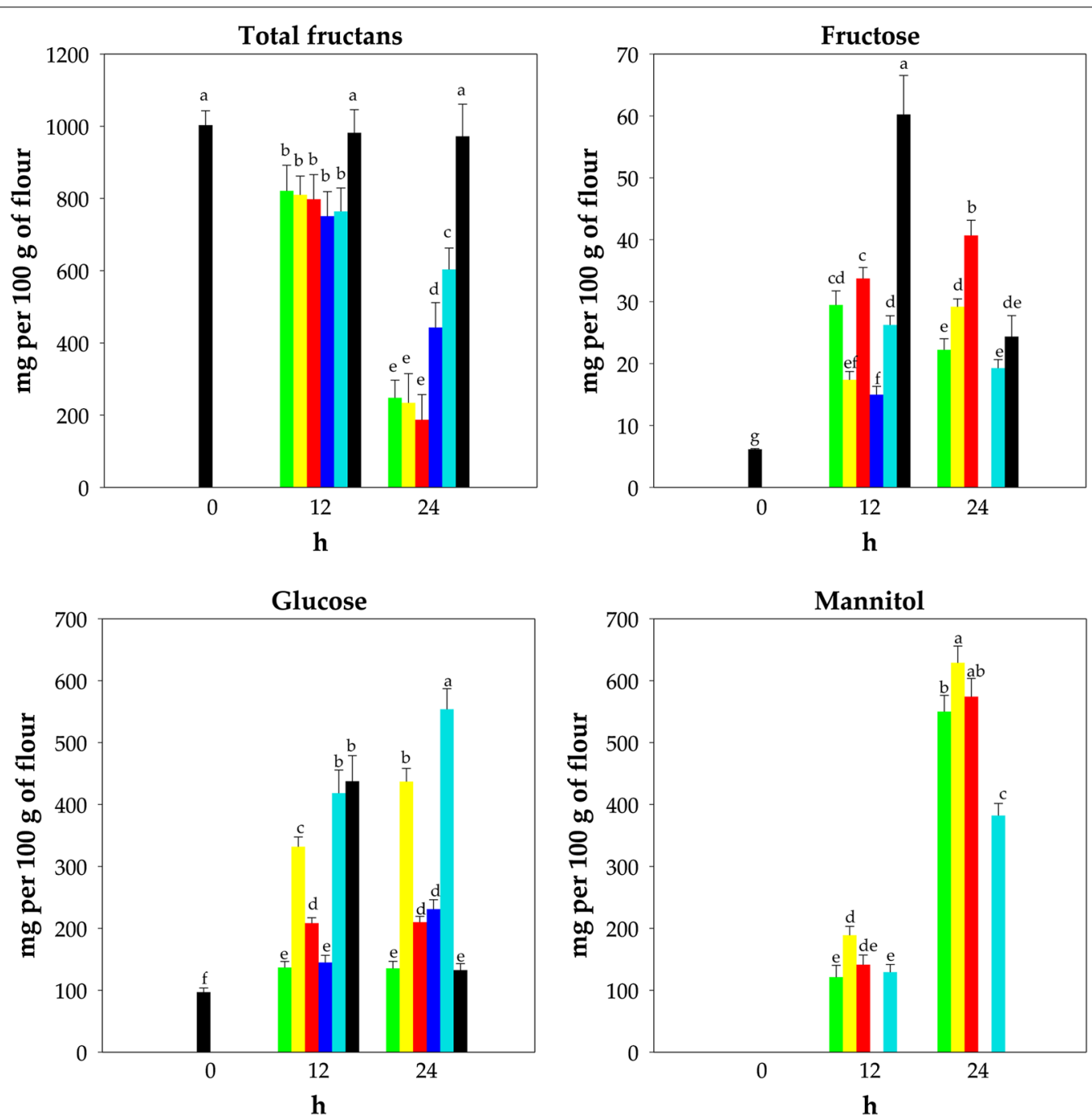

Fig. 2 Total fructans, fructose, glucose, and mannitol level (mg per $100 \mathrm{~g}$ of flour) in doughs. Wheat doughs (DY 160) were fermented with Apilactobacillus kunkeei B23I (green bars), Fructobacillus fructosus MBIII5 (yellow bars), A. kunkeei B23I + Fructo. fructosus MBIII5 (red bars), Lactiplantibacillus plantarum DC400 (blue bars) or Fructilactobacillus sanfranciscensis SD8 (cyan bars) at $30^{\circ} \mathrm{C}$ for $24 \mathrm{~h}$. A control dough without bacterial inoculum was incubated under the same conditions (black bars). Bars with different superscript letters (a-g) are significant different $(P<0.05)$ 
of fermentation with the two selected FLAB strains, the concentration of fructans significantly $(\mathrm{P}<0.05)$ decreased by $75-81 \%$ (Fig. 2). The drop $(\mathrm{P}<0.05)$ observed with L. plantarum DC400 and F. sanfranciscensis SD8 after the same time was of ca. $56 \%$ and $40 \%$, respectively (Fig. 2). No significant $(\mathrm{P}>0.05)$ change was found for the unstarted dough (Fig. 2).

Compared to the initial concentration of fructose $(6.2 \pm 0.1 \mathrm{mg}$ per $100 \mathrm{~g}$ of flour), an increasing release $(\mathrm{P}<0.05)$ was observed during fermentation with the selected strains (Fig. 2). After $24 \mathrm{~h}$ of fermentation by selected FLAB, the level of fructose increased to 22.2-40.7 mg per $100 \mathrm{~g}$ of flour (Fig. 2). Only L. plantarum DC400 was able to fully deplete fructose, whereas with $F$. sanfranciscensis SD8 the residual fructose was $19.3 \pm 1.4 \mathrm{mg}$ per $100 \mathrm{~g}$ of flour (Fig. 2). Fructose in excess of glucose was not found in any sample (Fig. 2). Mannitol was mainly released in doughs fermented with FLAB strains and, to a lesser extent, with $F$. sanfranciscensis SD8 (Fig. 2). After $24 \mathrm{~h}$, the highest amount of mannitol was detectable with Fructo. fructosus MBIII5 (629.1 $\pm 27.2 \mathrm{mg}$ per $100 \mathrm{~g}$ of flour) (Fig. 2). Mannitol was never detected in the dough fermented with L. plantarum DC400 and into the unstarted dough (Fig. 2).

\section{Protocol set up and validation}

Preliminary trials were carried out to define the best conditions in order to achieve the efficient enzymatic hydrolysis of fructans in wheat doughs (DY 160). The enzymatic treatment with invertase $\left(100 \mathrm{U} \mathrm{g}^{-1}\right.$ of substrates) at $35^{\circ} \mathrm{C}$ for $3 \mathrm{~h}$ was suitable to reach the hydrolysis of most of fructans (ca. 80\%). Combinations of FLAB and enzymatic treatment were performed in order to fully degrade FODMAPs in firm (DY 160) and liquid (DY 280) wheat doughs. Accordingly to the defined protocol, doughs were made by mixing invertase $\left(100 \mathrm{U} \mathrm{g}^{-1}\right.$ of substrates) and inoculating (ca. 8 Log CFU g ${ }^{-1}$ ) with single starter, composed by A. kunkeei B23I (Treatment 1, T1) or Fructo. fructosus MBIII5 (Treatment 2, T2), or mixed starters consisting of A. kunkeei B23I and Fructo. fructosus MBIII5 (Treatment 3, T3). A further dough was made and used as a control, which included the invertase and a mixed starter comprising L. plantarum DC400 and F. sanfranciscensis SD8 (Control treatment, CT). Doughs were fermented at $35{ }^{\circ} \mathrm{C}$ for $3 \mathrm{~h}$. Liquid doughs were incubated under aerobic condition in shaking $(200 \mathrm{rpm})$ flasks with baffles.

As shown in Figs. 3 and 4, the effect of treatments on FODMAPs level was mainly starter dependent, even though process parameters such DY and incubation conditions also contributed. Fructans were completely depleted in doughs fermented by FLAB (treatments $\mathrm{T} 1, \mathrm{~T} 2$, and T3) both under firm and liquid conditions.

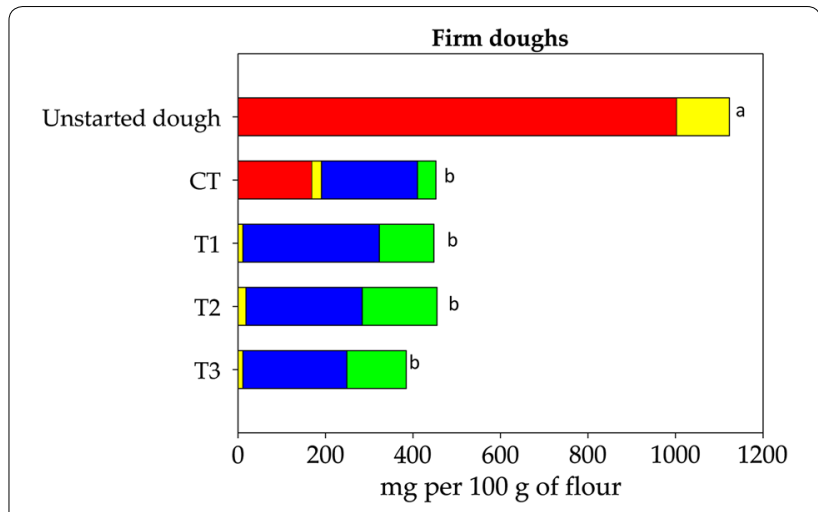

Fig. 3 FODMAPs (mg per $100 \mathrm{~g}$ of flour) in firm (DY 160) doughs. Wheat doughs were added with invertase (EC 3.2.1.26) and fermented at $35^{\circ} \mathrm{C}$ for $3 \mathrm{~h}$ with Apilactobacillus kunkeei B23l (Treatment 1,T1), or Fructobacillus fructosus MBIII5 (Treatment 2, T2), or with a mixed starter composed by A. kunkeei B23I and Fructo. fructosus MBIII5 (Treatment 3, T3), or Lactiplantibacillus plantarum DC400 and Fructilactobacillus sanfranciscensis SD8 (Control treatment, CT). Unstarted dough was also analyzed. Total fructans, red bars; galactosyl-sucrose oligosaccharides, yellow bars; fructose in excess of glucose, blue bars; mannitol, green bars. Bars with different superscript letters $(a-d)$ are significant different $(P<0.05)$

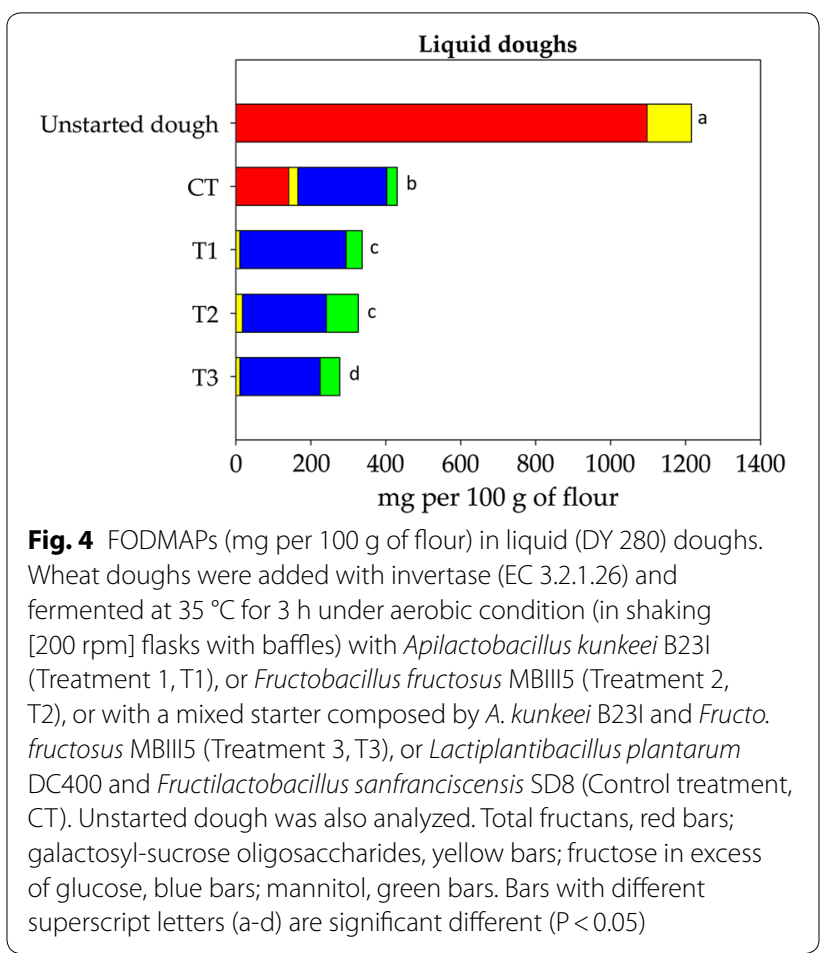

On the contrary, only $83-87 \%$ of fructans were hydrolyzed with CT (Figs. 3 and 4). Fructose in excess of glucose ranged from 214.2 to $311.9 \mathrm{mg}$ per $100 \mathrm{~g}$ of flour in doughs fermented by FLAB, and from 219.5 to $237.3 \mathrm{mg}$ 
per $100 \mathrm{~g}$ of flour in CT (Figs. 3 and 4). GOS underwent a reduction of $80-91 \%$ depending on the treatment (Figs. 3 and 4). Mannitol was mainly detectable in firm doughs subjected to T1, T2 and T3 (124.7-171.1 mg per $100 \mathrm{~g}$ of flour) (Fig. 3). Significantly lower $(\mathrm{P}<0.05)$ amount of mannitol were detectable in liquid doughs under the same treatments (43.2-85.2 $\mathrm{mg}$ per $100 \mathrm{~g}$ of flour) (Fig. 4). Mannitol levels in doughs subjected to CT were 42.2 and $28.0 \mathrm{mg}$ per $100 \mathrm{~g}$ of flour under firm and liquid conditions, respectively (Figs. 3 and 4). Considering the total amount of FODMAPs, no significant differences $(\mathrm{P}>0.05)$ were found between T1, T2, T3 and CT in firm doughs. On the contrary, fermentation of liquid doughs with FLAB led to lower $(\mathrm{P}<0.05)$ levels of FODMAPs compared to $\mathrm{CT}$. Overall, the lowest $(\mathrm{P}<0.05)$ amount of FODMAPs (277.8 mg per $100 \mathrm{~g}$ of flour) was reached with T3 under liquid conditions (Fig. 4).

\section{Discussion}

Graminan-type fructans are the main FODMAPs in wheat-based products, and their content may be reduced via bioprocessing with endogenous enzymes and/or microbial fermentation [1, 2, 9]. Sourdough fermentation should allow diminishing FODMAP levels $[9,15]$. The extent of reduction is dependent on the sourdough dosage, time of fermentation, type of flour, and, especially, the microbiota. Yeasts and few lactic acid bacteria species are able to hydrolyze fructans. During bioprocessing treatment on fructans, side reactions might lead to new FODMAPs. For instance, fructanase treatment releases large amounts of fructose, which in turn may be converted into mannitol throughout fermentation [9]. Therefore, our study investigated the complementary capability of targeted commercial enzymes and metabolically strictly FLAB to hydrolyze fructans and deplete fructose during wheat dough fermentation. FLAB prefer fructose as a growth substrate, because of its dual function as carbon source and external electron acceptor to achieve the $\mathrm{NAD}^{+} \mathrm{co}$-factor regeneration by the reduction of fructose to mannitol. Their growth on glucose depends on the availability of an additional electron acceptor, like fructose or oxygen [16-24]. Despite the evidence of their preferential consumption of fructose, little information is available on the biokinetics of FLAB during the growth in complex media or under food-like conditions. Our approach combined growth assays in synthetic media containing more than one sugar as substrate and fermentation trials in wheat doughs. During growth into a medium containing both fructose and glucose, FLAB strains displayed higher fructose consumption rate compared to conventional sourdough lactic acid bacteria such as L. plantarum and $F$. sanfranciscensis. Both fructophilic species A. kunkeei and Fructo. fructosus showed the same metabolic behavior although differences were found at strain level, with A. kunkeei B23I as the best performing. Fructose metabolism by FLAB was faster than glucose, regardless of the presence of oxygen, which can be used as external electron acceptor in alternative to fructose [16-24]. These preliminary findings suggested that fructose transport is privileged in FLAB over glucose, and supports their potential role in depletion of fructose resulting from fructans hydrolysis. Accordingly to the literature, we observed a very low fructose consumption rate by conventional sourdough lattobacilli used as controls [25]. In fact, fructose utilization by homo-fermentative and facultative hetero-fermentative species generally take place only after glucose depletion or for cell maintenance, whereas hetero-fermentative lactobacilli use fructose mostly as an electron acceptor. For instance, most of $F$. sanfranciscensis strains are unable to use fructose as carbon source, but generally display mannitol dehydrogenase activity and reduce fructose to mannitol $[25,26]$.

Because of the FLAB requirement to maintain their redox balance by reducing fructose into mannitol, this latter might accumulate during wheat dough fermentation. Therefore, we investigated the catabolism of mannitol with the goal of its reuse by FLAB. Only A. kunkeei B7 and five Fructo. fructosus strains were able to degrade mannitol, although its consumption rate was lower compared to fructose and glucose. Mannitol metabolism has been previously described in homo-fermentative lactobacilli as mediated by a mannitol-specific PTS system and a mannitol-1-phosphate-dehydrogenase [27, 28]. While FLAB have been previously shown to metabolize mannitol [16, 24], in this bacterial group the underling pathway and regulatory mechanism have not been rationalized. Based on our findings, this pathway is differently expressed by FLAB. Fructo. fructosus MBIII5 was the most active strain in mannitol consumption, leading to lactic and acetic acids as products.

Our mechanistic approach allowed the selection of two FLAB strains as starter candidates for low FODMAPs baking. A. kunkeei B23I was chosen for its high fructose consumption rate and Fructo. fructosus MBIII5 was selected for its ability to use mannitol as carbon source. Under sourdough condition, these two strains showed growth and acidification performances almost comparable to those of $L$. plantarum and $F$. sanfranciscensis strains. Anyway, the assessment of the degradation and consumption of saccharides during wheat dough fermentation revealed different metabolic features among different strains. Higher fructans breakdown occurred in FLAB inoculated doughs. Studies describing fructans degradation by FLAB are lacking. Only Prückler et al. [29] previously reported fructans breakdown in wheat 
bran fermented by Fructo. fructosus, but the same extent was observable with $F$. sanfranciscensis. Metabolism of fructans in lactobacilli is mediated by ATP-Binding-Cassette transport system, phosphoenolpyruvate-dependent phosphotranferase transport system ( $\beta$-glucoside family), and intracellular fructosidases [30]. In wheat flour, a large fraction of fructans exhibits a degree of polymerization (DP) ranging from 4 and 19, and the hydrolysis requires extracellular fructanases, which are infrequent in lactobacilli. Extracellular fructanases were characterized in Lactobacillus paracasei, and Lactobacillus crispatus [31, 32], and analogous DNA sequence of extracellular fructanases were identified in genome of few other species [9]. The different enzymatic pathways for fructans utilization by lactobacilli determine their abilities to degrade low- or high-DP fructans. Intracellular fructosidases limit the ability of lactobacilli to use only low-DP fructans $[9,30]$. Based on our evidences concerning the high hydrolysis capability by FLAB, their fructans utilization pathways deserve further investigation with the prospect of identifying enzymatic activities uncommon in lactobacilli. During wheat dough fermentation, the high fructose consumption rate by FLAB was confirmed, although the consumption was partly masked due to the release of free fructose during the decomposition of fructans, sucrose, and GOS. Both A. kunkeei B23I and Fructo. fructosus MBIII5 led to an increase in mannitol levels, despite the ability of Fructo. fructosus MBIII5 to degrade mannitol was shown into a synthetic medium. It is not unlikely that reutilization of mannitol would occur only after carbohydrate sources exhaustion and mannitol accumulation, due to induction of the mannitol transport system.

Preliminary trials allowed to develop a novel fermentation protocol exploiting the complementary capability of commercial invertase and selected FLAB strains for low FODMAPs baking. Fermentations were carried out using A. kunkeei B23I and Fructo. fructosus MBIII5 as single or binary starter both under firm- and liquiddough conditions. Overall, the use of binary starter to ferment liquid dough led to the lowest amount of FODMAPs (277.8 mg per $100 \mathrm{~g}$ of flour). Based on the proposed threshold value for single FODMAPs compound [7,33], the residual level in bread prepared according to our FLAB-based protocol was low enough to minimize symptom induction in FODMAPs sensitive people but guarantying a fiber intake comparable to regular bread. A low-FODMAPs rye bread with a total fructans content of $0.3 \mathrm{~g}$ per $100 \mathrm{~g}$ of brad was previously shown to reduce symptoms and gastrointestinal gas accumulation in IBS patients [33]. Our protocol did not exert the same positive effect on the total FODMAPs under firm-dough conditions, mainly due to the high accumulation of mannitol.
Likely, liquid dough fermentation under aerobic condition allowed higher oxygenation than in firm dough, resulting in lower mannitol production [28]. Secondarily, oxygen may have supported mannitol catabolism by Fructo. fructosus MBIII5, as the requirement for an external electron acceptor has been previously described for other lactobacilli during mannitol fermentation [34].

\section{Conclusions}

The results of our pioneering study clearly demonstrated the potential of selected strictly FLAB to strongly reduce FODMAPs in wheat dough, particularly under liquiddough and high oxygenation conditions. Although the best performances have been reached applying our protocol to liquid doughs, we have to point that the industrial demand for more efficient, controllable, largescale fermentation processes led to prefer type II sourdoughs, which are pumpable (semi-fluid) and consequently easy to handle. Our standardized protocol is also suitable for type III sourdough preparations.

Further investigation should be performed to evaluate potential synergistic interactions between FLAB and yeasts. Yeasts such as Saccharomyces cerevisiae and Kluyveromyces marxianus have proved their ability to degrade fructans [2] and therefore might contribute to reduce FODMAP levels in a complementary way with FLAB and commercial enzymes. Since FLAB have been found in association with yeasts in several ecological niches, we hypothesized the absence of antagonistic relationships between these two microbial groups, although specific negative interactions at strain or species level may not be excluded $[35,36]$.

Clinical trials will need to confirm the suitability for patients with IBS or non-celiac wheat sensitivity of wheat breads obtained according to our protocol.

\section{Methods \\ Microorganisms and culture conditions}

We used for fermentation 25 strains of strictly FLAB (Table 2). Previously, the strains isolation was from beegut (Apis mellifera L.) and ivy (Hedera helix L.) pollen [16, 35]. Lactiplantibacillus plantarum DC400 and Fructilactobacillus sanfranciscensis SD8 were the control strains (without preference of fructose over glucose as growth substrate), representing the conventional species of lactic acid bacteria mostly involved in sourdough fermentation [37]. All the strains belong to the Culture Collection of the Department of Soil, Plant and Food Science, University of Bari Aldo Moro (Bari, Italy), whose identification was by partial sequencing of the $16 S \mathrm{rRNA}$ and $r e c A$ genes. Cultures were maintained as stocks in $15 \%\left(\mathrm{v} \mathrm{v}^{-1}\right)$ glycerol at $-80{ }^{\circ} \mathrm{C}$ and routinely propagated. FLAB strains were grown in Fructose Yeast extract 
Table 2 Strictly FLAB strains $(n=25)$ used in this study

\begin{tabular}{lll}
\hline Strain & Source & Reference \\
\hline $\begin{array}{l}\text { Apilactobacillus kunkeei BIII59, BIII60, BVI14, B17, B23I, B7, BV61, BV20, BVI52, B4I } \\
\text { A. kunkeei BEE4 }\end{array}$ & $\begin{array}{l}\text { Apis mellifera L. bee-gut } \\
\text { A. mellifera L. bee-gut }\end{array}$ & 16 \\
& & $\begin{array}{c}\text { Filannino et al., } \\
\text { unpublished } \\
\text { data }\end{array}$ \\
A. kunkeei PLB29, PLA21, PFA3, PLB20, PLB 34, PL24, PF16, PF6 & Hedera helix L. pollen & 35 \\
Fructobacillus fructosus MBIII5, B5, MBIII2, B1 & A. mellifera L. bee-gut & 16 \\
Fructo. fructosus PL21, PFA23 & H. helix L. pollen & 35 \\
\hline
\end{tabular}

Peptone (FYP) broth (10 g D-fructose, $10 \mathrm{~g}$ yeast extract, $5 \mathrm{~g}$ polypeptone, $2 \mathrm{~g}$ sodium acetate, $0.5 \mathrm{~g}$ Tween $80,0.2 \mathrm{~g}$

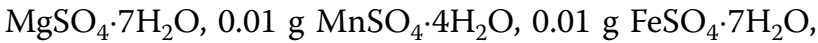
$0.01 \mathrm{~g} \mathrm{NaCl}$ per liter of distilled water $[\mathrm{pH} 6.8])$ at $30{ }^{\circ} \mathrm{C}$ until the late exponential growth phase was reached (ca. 18 h) [17]. L. plantarum DC400 and F. sanfranciscensis SD8 were grown in Sour Dough Bacteria (SDB) broth at $30{ }^{\circ} \mathrm{C}$ until the late exponential growth phase was reached (ca. 18 h) [38].

\section{Fructose consumption rate by FLAB into a fructose-glucose based medium}

All strains were screened for the determination of fructose consumption rate by using a fructose-glucose based medium. Cells were cultivated in FYP broth until the late exponential growth phase was reached (ca. $18 \mathrm{~h}$ ), harvested by centrifugation $(10,000 \times g$ for $10 \mathrm{~min}$ at $4{ }^{\circ} \mathrm{C}$ ), washed twice in $50 \mathrm{mM}$ phosphate buffer, $\mathrm{pH} 7.0$, and re-suspended at the initial cell density of ca. 7 Log CFU mL $\mathrm{mL}^{-1}$ into a Fructose Glucose Yeast extract Peptone (FGYP) broth (identical to FYP broth but added with glucose, $\left.1 \% \mathrm{w} \mathrm{v}^{-1}\right)$. Then, cells were incubated aerobically at $30{ }^{\circ} \mathrm{C}$ for $24 \mathrm{~h}$. Supernatants were recovered from FGYP cultures every $2 \mathrm{~h}$ for $24 \mathrm{~h}$, centrifuged at $(10,000 \times g$ for $10 \mathrm{~min}$ at $4{ }^{\circ} \mathrm{C}$ ), filtered through 0.22 - $\mu \mathrm{m}$-pore-size filter (Millipore), and used to determine glucose and fructose by high-performance liquid chromatography (HPLC). An Äkta purifier system (GE Healthcare) equipped with an Aminex HPX-87H column (ion exclusion; Bio-Rad), and a PerkinElmer 200a refractive index detector (PerkinElmer, Waltham, MA) operating at $32{ }^{\circ} \mathrm{C}$ were used. Elution was at $60{ }^{\circ} \mathrm{C}$ with a flow rate of $0.4 \mathrm{ml} \mathrm{min}^{-1}$, and $10 \mathrm{mmol} \mathrm{l}^{-1} \mathrm{H}_{2} \mathrm{SO}_{4}$ was used as mobile phase [39]. Commercial standards (Sigma-Aldrich; Merck KGaA, Darmstadt, Germany) allowed the identification and quantification of each compounds. Kinetics of fructose consumption were determined and modeled according to the Gompertz equation as modified by Zwietering et al. [14]:

$$
y=k+D \exp \left(-\exp \left(\left(\left(\mu_{\max } \times 2.7182\right)(\lambda-t) / D\right)+1\right)\right) .
$$

where $\mathrm{k}$ is the initial level of fructose (expressed as $\mathrm{g}^{-1}$ ), $D$ is the highest amount of fructose consumed (expressed as $\mathrm{g}^{-1}$ ) at the stationary phase, $\mu_{\max }$ is maximum consumption rate (expressed as $\mathrm{g}^{-1} \mathrm{~h}^{-1}$ ), $\lambda$ is the length of the lag phase expressed in hours, and $t$ is the time. Experimental data were modelled by the non-linear regression procedure of the Statistica 8.0 software (Statsoft, Tulsa, USA).

\section{Mannitol consumption by FLAB in mannitol-based media}

FLAB strains were sub-cultured in modified FYP broth containing fructose $\left(0.5 \% \mathrm{w} \mathrm{v}^{-1}\right)$ and mannitol $\left(1 \% \mathrm{w} \mathrm{v}^{-1}\right)$ as carbon sources. Sub-cultured cells were harvested, washed as described above, and inoculated on Mannitol Yeast extract Peptone (MYP) agar (identical to FYP medium but fructose was replaced by mannitol) supplemented with $0.5 \% \mathrm{CaCO}_{3}\left(\mathrm{w} \mathrm{v}^{-1}\right)$. Plates were incubated at $30{ }^{\circ} \mathrm{C}$ for $48 \mathrm{~h}$. Mannitol consumption was evaluated by measuring the size of the clearance zone surrounding the colonies, which indirectly indicates the hydrolysis of $\mathrm{CaCO}_{3}$ reacting with organic acids synthesized by bacteria.

Mannitol consumption and deriving metabolites were also investigated during FLAB growth in MYP broth. Cells were cultivated in FYP broth until the late exponential growth phase was reached (ca. $18 \mathrm{~h}$ ), harvested as described above, and re-suspended at the initial cell density of ca. 7 Log CFU mL ${ }^{-1}$ into a MYP broth. Cells were cultured at $30{ }^{\circ} \mathrm{C}$ for $72 \mathrm{~h}$ under aerobic condition in shaking $(200 \mathrm{rpm})$ flasks with baffles. Organic acids and mannitol concentrations were determined by HPLC as described above, using external standards (SigmaAldrich). The $\mathrm{pH}$ was measured by a Crison $\mathrm{pH}$-meter (Model 507, Crison, Milan, Italy).

\section{Model-sourdough fermentation}

Commercial durum wheat (Triticum durum) flour was purchased from a local market in Italy. Gross composition was as follows: moisture, $12.6 \pm 0.42 \%$; protein $(\mathrm{N} \times 5.7), 11.9 \pm 0.01 \%$ of dry matter (d.m.); total 
carbohydrates, $70.9 \pm 0.3 \%$ of d.m.; fat $1.0 \pm 0.01 \%$ of d.m. Flour (187.5 g) and tap water $(112.5 \mathrm{ml})$ were used to produce $300 \mathrm{~g}$ of dough (dough yield [dough weight $\times 100$ /flour weight], 160) with a continuous high-speed mixer $(60 \times \mathrm{g}$, dough mixing time of $5 \mathrm{~min})$ (Chopin \& Co., Boulogne, Seine, France).

Pure culture of selected A. kunkeei B23I and Fructo. fructosus MBIII5 strains were used singly or as mixed starters. The mixed starter consisted of binary combinations including two bacterial strains inoculated at the same cell density (ca. 7 Log CFU g ${ }^{-1}$ ). Positive interactions among strains or species of lactic acid bacteria have been described by several studies [40]. Thus, in this study we proposed the combination of $A$. kunkeei B23I with Fructo. fructosus MBIII5 to exploit complementarily their metabolic traits, which were highlighted during the preliminary trials. L. plantarum DC400 and F. sanfranciscensis SD8 were used as control starters. Cells were cultivated in FYP or SDB broths and inoculated into the dough at the initial cell density of ca. 7 Log CFU g ${ }^{-1}$, as described above. A control dough was prepared with durum wheat flour and sterile tap water, without bacterial inoculums (unstarted dough). Doughs were incubated for $24 \mathrm{~h}$ at $30{ }^{\circ} \mathrm{C}$. Acidification and bacterial growth were monitored every $12 \mathrm{~h}$ for $72 \mathrm{~h}$. The $\mathrm{pH}$ value of doughs was determined by a $\mathrm{pH}$-meter with a food penetration probe. Microbiological analyses were carried out on $10 \mathrm{~g}$ of dough homogenized with $90 \mathrm{ml}$ of sterile peptone water (peptone $1 \% \mathrm{wt} / \mathrm{vol}$ and $\mathrm{NaCl} 0.9 \% \mathrm{wt} / \mathrm{vol}$ ) solution. Presumptive lactic acid bacteria were enumerated by plating on FYP agar or SDB agar. Media were supplemented with cycloheximide $\left(0.1 \mathrm{~g} \mathrm{~L}^{-1}\right)$. Plates were incubated at $30{ }^{\circ} \mathrm{C}$ for $48 \mathrm{~h}$.

\section{Determination of total fructans, fructose, glucose, and mannitol levels in fermented doughs}

Levels of total fructans, fructose, glucose, and mannitol were monitored every $12 \mathrm{~h}$ during fermentation. Samples were freeze-dried and grinded before analyses.

Glucose, fructose and mannitol were quantified by HPLC as described above. Prior the analysis, $10 \mathrm{~mL}$ of perchloric acid $\left(5 \%, \mathrm{v} \mathrm{v}^{-1}\right)$ were added to $10 \mathrm{~g}$ of sample as precipitating agent. Then, the suspension was kept overnight at $4{ }^{\circ} \mathrm{C}$, centrifuged at $10,000 \times g$ for $10 \mathrm{~min}$, and filtered through a $0.22-\mathrm{mm}$ pore size filter.

Total fructans were measured using the Fructan Assay Kit (Megazyme International Ireland Ltd) as per manufacturer's instructions. The extraction process was carried out as described by Muir et al. [41], with few changes. One $\mathrm{g}$ of freeze-dried dough was milled and mixed with $40 \mathrm{ml}$ of hot distilled water $\left(80^{\circ} \mathrm{C}\right)$, and stirred on a hot-plate $\left(80{ }^{\circ} \mathrm{C}\right)$ for $15 \mathrm{~min}$, until the sample is completely dispersed. Then, sample was cooled at room temperature and the volume was adjusted to $50 \mathrm{ml}$ with distilled water.

\section{Enzymes}

Endo-inulinase from Aspergillus niger (EC 3.2.1.7), exoinulinase from $A$. niger (EC 3.2.1.80), and invertase (fructofuranosidase) from yeasts (EC 3.2.1.26) were purchased from Megazyme International Ireland Ltd. The enzyme activities were investigated during preliminary trials carried out on wheat flour doughs (DY 160) under different concentrations (10-100 $\mathrm{U} \mathrm{g}^{-1}$ of substrates), temperatures $\left(30-37^{\circ} \mathrm{C}\right)$, and time of incubation $(2-4 \mathrm{~h})$ in order to define the best conditions of use. Fructans hydrolysis was monitored by HPLC analysis and the Fructan Assay Kit (Megazyme International Ireland Ltd) as described above.

\section{Protocol set up and validation}

Based on the preliminary experiment results, further fermentation trials were carried out on firm (DY, 160) and liquid (DY, 280) wheat doughs. Doughs were mixed with invertase (100 $\mathrm{U} \mathrm{g}^{-1}$ of substrates) and inoculated at a cell density of ca. $8 \mathrm{Log}_{\mathrm{CFU} \mathrm{g}}{ }^{-1}$ with a starter composed by $A$. kunkeei B23I (Treatment 1, T1) or Fructo. fructosus MBIII5 (Treatment 2, T2), or a mixed starter composed by a combination of $A$. kunkeei $\mathrm{B} 23 \mathrm{I}$ and Fructo. fructosus MBIII5 (Treatment 3, T3). A further condition was used as a control combining the enzymatic treatment with a mixed starter composed of L. plantarum DC400 and $F$. sanfranciscensis SD8 (Control treatment, CT). The mixed starters consisted of binary combinations of two bacterial strains both inoculated at a cell density of ca. $8 \mathrm{Log}$ $\mathrm{CFU} \mathrm{g}{ }^{-1}$. Doughs were fermented at $35^{\circ} \mathrm{C}$ for $3 \mathrm{~h}$. Liquid doughs were incubated under aerobic condition in shaking $(200 \mathrm{rpm})$ flasks with baffles.

Levels of total fructans, fructose, glucose, and mannitol were determined as described above. GOS were measured using an enzymatically based assay kit (Raffinose/DGalactose Assay Kit, Megazyme International Ireland Ltd), according to the manufacturer's instructions.

\section{Statistical analysis}

All the fermentations were in triplicate and samples underwent analysis in triplicate. Data underwent to oneway ANOVA by Statistica for Windows (Statistica7.0 per Windows). Tukey's test was used to determine significant differences among means at an error probability of $5 \%$ $(\mathrm{P}<0.05)$. 


\section{Abbreviations}

FODMAPs: Fermentable oligosaccharides, disaccharides, monosaccharides, and polyols; FLAB: Fructophilic lactic acid bacteria; GOS: Galacto-oligosaccharides; IBS: Irritable bowel syndrome; FYP: Fructose Yeast extract Peptone; FGYP: Fructose Glucose Yeast extract Peptone; MYP: Mannitol Yeast extract Peptone; T1: Treatment 1;T2: Treatment 2; T3: Treatment 3; CT: Control treatment.

\section{Acknowledgements}

Not applicable.

\section{Authors' contributions}

RDC, PF and MG conceived the idea. RDC and PF designed the experiments. $\mathrm{RDC}$ and $M G$ were the supervisors and the coordinators of the research units. MAA, PF and VC carried out the experiments. MAA and PF analyzed the data and wrote the original manuscript draft. RDC and MG reviewed the manuscript. All authors have read and agreed to the published version of the manuscript. All authors read and approved the final manuscript.

\section{Funding}

The article-processing charges were funded by the Open Access Publishing Fund of the Free University of Bozen-Bolzano.

\section{Availability of data and materials \\ The data sets supporting the conclusions of this article are included within the article.}

\section{Ethics approval and consent to participate}

Not applicable.

\section{Consent for publication}

Not applicable.

\section{Competing interests}

The authors declare that they have no competing interests.

Received: 12 July 2020 Accepted: 9 September 2020

Published online: 17 September 2020

\section{References}

1. Yan YL, Hu Y, Gänzle MG. Prebiotics, FODMAPs and dietary fiber-conflicting concepts in development of functional food products? Curr Opin Food Sci. 2018:20:30-7.

2. Menezes LA, Minervini F, Filannino P, Sardaro ML, Gatti M, Lindner JDD. Effects of sourdough on FODMAPs in bread and potential outcomes on irritable bowel syndrome patients and healthy subjects. Front Microbiol. 2018;9:1972.

3. De Giorgio R, Volta U, Gibson PR. Sensitivity to wheat, gluten and FODMAPs in IBS: facts or fiction? Gut. 2016;65:169-78.

4. Schuppan D, Pickert G, Ashfaq-Khan M, Zevallos V. Non-celiac wheat sensitivity: differential diagnosis, triggers and implications. Best Pract Res Clin Gastroenterol. 2015;29:469-76.

5. Liu J, Chey WD, Haller E, Eswaran S. Low-FODMAP diet for irritable bowel syndrome: what we know and what we have yet to learn. Annu Rev Med. 2020;71:303-14.

6. Biesiekierski JR, Peters SL, Newnham ED, Rosella O, Muir JG, Gibson PR. No effects of gluten in patients with self-reported non-celiac gluten sensitivity after dietary reduction of fermentable, poorly absorbed, short-chain carbohydrates. Gastroenterology. 2013;145:320-8.

7. Varney J, Barrett J, Scarlata K, Catsos P, Gibson PR, Muir JG. FODMAPs: food composition, defining cutoff values and international application. J Gastroenterol Hepatol. 2017;32:53-61.

8. Catassi G, Lionetti E, Gatti S, Catassi C. The low FODMAP diet: many question marks for a catchy acronym. Nutrients. 2017;9:292.

9. Loponen J, Gänzle MG. Use of sourdough in low FODMAP baking. Foods. 2018;7:96.

10. Nyyssölä A, Ellilä S, Nordlund E, Poutanen K. Reduction of FODMAP content by bioprocessing. Trends Food Sci Technol. 2020;99:257-72.
11. Gibson PR, Newnham E, Barrett JS, Shepherd SJ, Muir JG. Fructose malabsorption and the bigger picture. Aliment Pharmacol Ther. 2007;25:349-63.

12. Filannino P, Di Cagno R, Tlais AZA, Cantatore V, Gobbetti M. Fructose-rich niches traced the evolution of lactic acid bacteria toward fructophilic species. Crit Rev Microbiol. 2019;45:65-81.

13. Di Cagno R, Pontonio E, Buchin S, De Angelis M, Lattanzi A, Valerio F, Gobbetti M, Calasso M. Diversity of the lactic acid bacterium and yeast microbiota in the switch from firm-to liquid-sourdough fermentation. Appl Environ Microbiol. 2014;80:3161-72.

14. Zwietering MH, Jongenburger I, Rombouts FM, Van't Riet K. Modeling of the bacterial growth curve. Appl Environ Microbiol. 1990;56:1875-81.

15. Ziegler JU, Steiner D, Longin CFH, Würschum T, Schweiggert RM, Carle R. Wheat and the irritable bowel syndrome-FODMAP levels of modern and ancient species and their retention during bread making. J Funct Foods. 2016;25:257-66.

16. Filannino P, Di Cagno R, Addante R, Pontonio E, Gobbetti M. Metabolism of fructophilic lactic acid bacteria isolated from the Apis mellifera $\mathrm{L}$. bee gut: Phenolic acids as external electron acceptors. Appl Environ Microbiol. 2016. 82:6899-6911.

17. Endo A, Futagawa-Endo Y, Dicks LM. Isolation and characterization of fructophilic lactic acid bacteria from fructose-rich niches. Syst Appl Microbiol. 2009;32:593-600

18. Endo A, Okada S. Reclassification of the genus Leuconostoc and proposals of Fructobacillus fructosus gen. nov., comb. nov., Fructobacillus durionis comb. nov., Fructobacillus ficulneus comb. nov. and Fructobacillus pseudoficulneus comb. nov. Int J Syst Evol Microbiol. 2008. 58:2195-2205.

19. Endo A, Irisawa T, Futagawa-Endo Y, Takano K, du Toit M, Okada S, Dicks LM. Characterization and emended description of Lactobacillus kunkeei as a fructophilic lactic acid bacterium. Int J Syst Evol Microbiol. 2012:62:500-4.

20. Endo A, Tanaka N, Oikawa Y, Okada S, Dicks L. Fructophilic characteristics of Fructobacillus spp. may be due to the absence of an alcohol/acetaldehyde dehydrogenase gene (adhE). Curr Microbiol 2014. 68:531-535.

21. Endo A, Tanizawa Y, Tanaka N, Maeno S, Kumar H, Shiwa Y, Okada S, Yoshikawa H, Dicks L, Nakagawa J, Arita M. Comparative genomics of Fructobacillus spp. and Leuconostoc spp. reveals niche-specific evolution of Fructobacillus spp. BMC genomics. 2015. 16:1117.

22. Neveling DP, Endo A, Dicks LM. Fructophilic Lactobacillus kunkeei and Lactobacillus brevis isolated from fresh flowers, bees and beehives. Curr Microbiol. 2012;65:507-15.

23. Maeno S, Tanizawa Y, Kanesaki Y, Kubota E, Kumar H, Dicks L, Salminen S, Nakagawa J, Arita M, Endo A. Genomic characterization of a fructophilic bee symbiont Lactobacillus kunkeei reveals its niche-specific adaptation. Syst Appl Microbiol. 2016;39:516-26.

24. Maeno S, Kajikawa A, Dicks L, Endo A. Introduction of bifunctional alcohol/acetaldehyde dehydrogenase gene (adhE) in Fructobacillus fructosus settled its fructophilic characteristics. Res Microbiol. 2019;170:35-42.

25. Gänzle MG, Vermeulen N, Vogel RF. Carbohydrate, peptide and lipid metabolism of lactic acid bacteria in sourdough. Food Microbiol. 2007:24:128-38.

26. Neysens P, De Vuyst L. Kinetics and modelling of sourdough lactic acid bacteria. Trends Food Sci Technol. 2005:16:95-103.

27. Neves AR, Ramos A, Shearman C, Gasson MJ, Santos H. Catabolism of mannitol in Lactococcus lactis MG1363 and a mutant defective in lactate dehydrogenase. Microbiology. 2002;148:3467-76.

28. Wisselink HW, Moers AP, Mars AE, Hoefnagel MH, De Vos WM, Hugenholtz J. Overproduction of heterologous mannitol 1-phosphatase: a key factor for engineering mannitol production by Lactococcus lactis. Appl Environ Microbiol. 2005;71:1507-14.

29. Prückler M, Lorenz C, Endo A, Kraler M, Dürrschmid K, Hendriks K, Soares da Silva, F, Auterith E, Kneifel W, Michlmayr H. Comparison of homo-and heterofermentative lactic acid bacteria for implementation of fermented wheat bran in bread. Food Microbiol 2015. 49:211-219.

30. Zúñiga M, Yebra MJ, Monedero V. Complex oligosaccharide utilization pathways in Lactobacillus. Curr Issues Mol Biol. 2020;40:49.

31. Goh YJ, Lee JH, Hutkins RW. Functional analysis of the fructooligosaccharide utilization operon in Lactobacillus paracasei 1195. Appl Environ Microbiol. 2007;73:5716-24.

32. Loponen J, Mikola M, Sibakov J. An Enzyme Exhibiting Fructan Hydrolase Activity. Patent No. WO2017220864A1, 28 December 2017. 
33. Laatikainen R, Koskenpato J, Hongisto SM, Loponen J, Poussa T, Hillilä M, Korpela R. Randomised clinical trial: low-FODMAP rye bread vs. regular rye bread to relieve the symptoms of irritable bowel syndrome. Aliment Pharmacol Ther. 2016. 44:460-470.

34. McFeeters RF, Chen KH. Utilization of electron acceptors for anaerobic mannitol metabolism by Lactobacillus plantarum. Compounds which serve as electron acceptors. Food Microbiol. 1986:3:73-81.

35. Di Cagno R, Filannino P, Cantatore V, Gobbetti M. Novel solid-state fermentation of bee-collected pollen emulating the natural fermentation process of bee bread. Food Microbiol. 2019;82:218-30.

36. De Vuyst L, Weckx S. The cocoa bean fermentation process: from ecosystem analysis to starter culture development. J Appl Microbiol. 2016;121(1):5-17.

37. Gobbetti M, Corsetti A, Rossi J. The sourdough microflora. Interactions between lactic acid bacteria and yeasts: metabolism of carbohydrates. Appl Microbiol Biotechnol. 1994.41:456-460.

38. Kline L, Sugihara TF. Microorganisms of the San Francisco sour dough bread process: II. Isolation and characterization of undescribed bacterial species responsible for the souring activity. Appl Environ Microbiol. 1971. 21:459-465.

39. Zeppa G, Conterno L, Gerbi V. Determination of organic acids, sugars, diacetyl, and acetoin in cheese by high-performance liquid chromatography. J Agric Food Chem. 2001;49:2722-6.

40. Canon F, Nidelet T, Thierry A, Gagnaire V. Understanding the mechanisms of positive microbial interactions that benefit lactic acid bacteria cocultures. Front Microbiol. 2020;11:2088.

41. Muir JG, Rose R, Rosella O, Liels K, Barrett JS, Shepherd SJ, Gibson PR. Measurement of short-chain carbohydrates in common Australian vegetables and fruits by high-performance liquid chromatography (HPLC). J Agric Food Chem. 2009;57:554-65.

\section{Publisher's Note}

Springer Nature remains neutral with regard to jurisdictional claims in published maps and institutional affiliations.
Ready to submit your research? Choose BMC and benefit from:

- fast, convenient online submission

- thorough peer review by experienced researchers in your field

- rapid publication on acceptance

- support for research data, including large and complex data types

- gold Open Access which fosters wider collaboration and increased citations

- maximum visibility for your research: over $100 \mathrm{M}$ website views per year

At BMC, research is always in progress.

Learn more biomedcentral.com/submissions 